\title{
EINE NOTIZ ÜBER SCHREIBENDE UND WANDERNDE PHILOSOPHEN
}

\section{Fabia Neuerburg}

Universität Trier, Universitätsring 15, 54296 Trier, Deutschland; neuerburgf@uni-trier.de

In a letter allegedly written by Thales to Pherekydes, which is contained in the 'life of Thales' by Diogenes Laertius, the reader comes across a peculiar differentiation between two kinds of sages: Philosophers who travel the world but barely write anything down, thus resembling modern-day tourists, as opposed to philosophers who are devoted to their writings, yet do not travel at all. Could it be, that movement, i.e. travelling and wandering, is not compatible with being a true philosopher after all, as suggested by Silvia Montiglio? By revisiting the relevant paragraphs this note aims to examine whether this postulated irreconcilability has anything to do with the differentiation presented in the letter of Thales. A closer analysis of the text suggests that the underlying idea of this passage consists of two different concepts of theoria: a more traditional theoria, a journey in pursuit of knowledge, in contrast to a philosophical theoria, appropriated and transformed by Greek philosophers in the fourth century. Refs 8 .

Keywords: Diogenes Laertius, Thales, Pherekydes, travel, theoria.

In den Philosophenviten des Diogenes Laertius schreibt Thales in einem Brief an Pherekydes folgenden Vorschlag: Er, Thales, werde zu Pherekydes nach Syros reisen um dort mit diesem über die theologische Abhandlung zu sprechen, an welcher Pherekydes arbeite. Wenn Pherekydes es erlaube, werde auch Solon zu ihm kommen. ${ }^{1}$ Schließlich

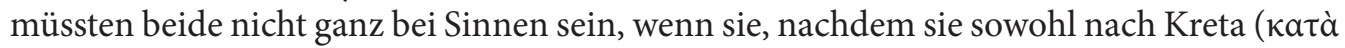

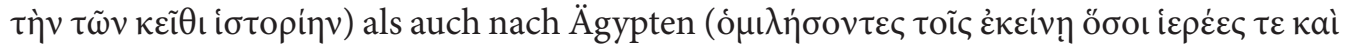

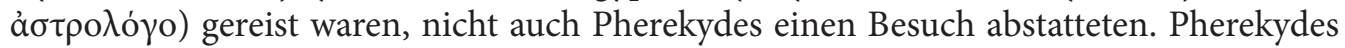
aber, der an seiner Heimat hänge ( $\chi \omega \rho \circ \varphi \iota \lambda \dot{\varepsilon} \omega v)$, besuche Ionien nur selten. Keine „Sehnsucht nach fremden Menschen" ( $\pi 0 \theta \grave{\eta} \alpha \dot{\alpha} \delta \rho \tilde{\omega} v \xi \xi i v \omega v)$ dränge ihn in die Welt hinaus. Stattdessen sei er ganz dem Schreiben hingegeben. „Wir aber, die nicht schreiben, bereisen ganz Griechenland und Asien“.

${ }^{1}$ Diog. Laert. 1, 43-44. Es ist nicht davon auszugehen, dass in dieser Stelle von einer gemeinsamen Reise des Thales und Solon die Rede ist. Vielmehr ist wohl gemeint, dass beide aus ihren jeweiligen Heimatstädten Milet und Athen nach Syros aufbrechen sollten. Eventuell diente gerade die geographische Lage der Insel, die zwischen den beiden Städten gelagert ist, als Inspirationsquelle für den Briefwechsel. Ich danke Michail Pozdnev für seine Anmerkungen zu dieser Stelle.

(C) St. Petersburg State University, 2017 
Die Reisenden, die nicht schreiben und die Schreibenden, die nicht reisen, scheinen hier eigentümlich unterschieden zu werden. Diogenes Laertius weist in der Thales-Vita natürlich darauf hin, dass dieser zwar weite Reisen unternommen, jedoch nichts Schriftliches hinterlassen habe. ${ }^{2}$ Hiermit befindet er sich in bester Gesellschaft mit dem notorischen Weltreisenden Pythagoras, dessen Reisen ihn mit fortschreitender Legendenbildung bis an das Ende der bekannten Welt führten und welcher ebenfalls keine Schriften überliefert. Diese Darstellung, ob historisch wahrscheinlich oder nicht, scheint zumindest auf den ersten Blick widersprüchlich: Warum langwierige und ausgiebige Reisen in oftmals exotische Länder unternehmen, wenn die gewonnenen Kenntnisse nicht in Schriften verbreitet werden? Wird die Unterscheidung in dieser Stelle deshalb betont, weil das Reisen, Bewegung überhaupt, als unbeständiger und unsteter Zustand per se nicht mit dem ernsthaften Philosophieren vereinbar und damit in diesem Kontext negativ konnotiert war? Silvia Montiglio stellt fest, dass das Reisen zumindest in frühester Zeit als nicht erstrebenswerter Zustand angesehen wurde, da es mit einem heimatlosen Umherziehen in Zusammenhang gebracht wurde. ${ }^{3}$ Noch bei Platon findet sie diese negative Einstellung seinen eigenen Reisen gegenüber, die allenfalls Mittel zum Zweck darstellen und keinesfalls förderlich für die Betätigung als Philosoph seien. Selbst in Bezug auf den Spaziergang während der philosophischen Diskussion weist sie darauf hin, dass dieser in den platonischen Dialogen teilweise nur einen anfänglichen Rahmen bildet. Sobald die eigentliche Untersuchung beginnt, kommt die Bewegung zum Erliegen: Die Figuren erreichen einen bestimmten Ort oder nehmen Platz, wie beispielsweise im Protagoras. ${ }^{4}$ Auch die philosophische Kontemplation des Einzelnen findet Montiglio zufolge eher im Stehen statt. In der von ihr herangezogenen Stelle aus dem Symposion bleibt Sokrates, während er mit Aristodemos und Agathon unterwegs ist, einfach in Gedanken versunken stehen. Sein Verhalten, das zunächst auf Unverständnis stößt, muss allerdings von Aristodemos als „eine seiner Angewohnheiten“5 erklärt werden und bleibt damit eher ein für Sokrates typisches Kuriosum, das schwerlich für „den Philosophen“ im Allgemeinen gelten kann.

Unzweifelhaft ist, dass das Umherwandeln, also die Bewegung, das langsame Gehen, untrennbar mit der philosophischen Diskussion verbunden ist. Von entscheidender Bedeutung ist hierbei jedoch ein gemäßigtes Tempo, welches allein dem Philosophen angemessen war und jeden Anschein von Hektik zurückwies. ${ }^{6}$ Die enge Verbindung von

${ }^{2} \mathrm{Ob}$ Thales überhaupt Schriften hinterließ ist schon in der Antike stark umstritten. Auch besteht Unklarheit über Anzahl und Titel möglicher Werke. Als Erster äußert sich Vitruv über Thales' Schriften, der jedoch nur ausgearbeitete Theorien naturphilosophischen Inhalts erwähnt (De arch. 9, 6, 3). Diogenes Laertius berichtet von einigen Autoren, die der Meinung seien, Thales habe nichts hinterlassen, da die

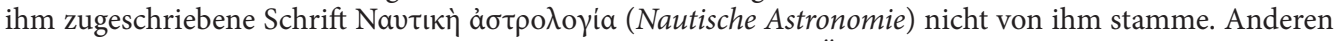

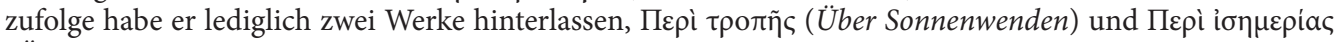
(Über Tag- und Nachtgleichen), (1,23). Damit nennt er alle drei namentlich bekannten, möglichen Werke des Thales. Zu den beiden letzteren Werken siehe Mejer 2002, 255-256. Die Philosophie des Thales ist hauptsächlich durch Aristoteles überliefert. Welche Quellen Aristoteles wiederum zur Verfügung standen ist nicht sicher: Betegh 2002.

${ }^{3}$ Montiglio 2000, 86-105.

${ }^{4}$ Pl. Prt. 317d.

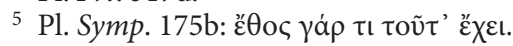

${ }^{6}$ Bremmer 1991, 18-20. Auch Aristoteles zufolge zeichnet sich der $\mu \varepsilon \gamma \alpha \lambda$ ó $\psi v \chi 0 \varsigma$, neben anderen Eigenschaften wie einer tiefen Stimme, unter anderem durch seinen langsamen Gang aus, Eth.Nic. 4, 3, 34 (1125a). 
philosophischem Gespräch und Bewegung setzt sich in römischer Zeit in der ambulatio fort, die neben der Imitation griechischer Tradition zusätzlich zur Demonstration aristokratischer Muße wird. ${ }^{7}$ Die Bemerkung bei Diogenes Laertius hat wohl wenig damit zu tun, dass Reisen durch ihren unsteten Charakter nicht mit Philosophie vereinbar sind. Vielmehr geht es um verschiedene Arten des Erkenntnisgewinns. Was Pherekydes angeblich abhält, ist seine „Heimatliebe“, die übersetzt den Drang ferne Länder zu bereisen, überwiegt, und sein Mangel an "Sehnsucht nach fremden Menschen“. Damit mutet die hier herrschende Vorstellung von Reisen fast schon touristisch an. Primär geht es um Erkenntnisgewinn durch das Erleben und „Sehen“, also eine theoria, wie sie zuerst bei Herodot in Erscheinung tritt. ${ }^{8}$ Dieser lässt Solon „der theoria wegen“ fremde Länder bereisen, wobei der theoria-Begriff sich hier bereits von seinem ursprünglich sakralen Kontext gelöst hat und zu „Erkenntnis, die auf Reisen gewonnen wird“ geworden ist. Der Reisebegriff in der Thalesvita bewegt sich damit ganz im Sinne der herodoteischen, profanierten theoria, bevor diese im 4. Jh. dann auf eine neue, metaphorische Ebene gehoben wurde.

\section{Literaturhinweise}

Betegh G. Thales. DNP, 12/1, Stuttgart - Weimar, 2002, Sp. 236-238.

Bremmer J. Walking, standing, and sitting in ancient Greek Culture, in: J. Bremmer, H. Roodenburg (Hgg.)

A Cultural History of Gesture. Cambridge, 1991, 15-35.

Mejer J. Eudemus and the History of Science, in: I. Bodnár, W. W. Fortenbaugh (Hgg), Eudemus of Rhodes. New Brunswick: Action Publishers, 2002, 243-261.

Montiglio S. Wandering Philosophers in ancient Greece. JHS 2000, 120, 86-105.

Nightingale A. W. Spectacles of Truth in Classical Greek Philosophy. Theoria in its Cultural Context. Cambridge, Cambridge University Press, 2004.

O'Sullivan T. Walking in Roman Culture, Cambridge, Cambridge University Press, 2011.

Rausch H. Theoria. Von ihrer sakralen zur philosophischen Bedeutung, München, Fink, 1982.

Sassi M. M. Il viaggio e la festa. Note sulla rappresentazione dell'ideale filosofico della vita, in: G. Camassa, S. Fasce (Hgg.), Idea e realtà del viaggio. Il viaggio nel mondo antico. Genova 1991, 17-36.

For citation: Fabia Neuerburg. Eine Notiz über schreibende und wandernde Philosophen. Philologia Classica 2017, 12(1), 111-114. DOI: 10.21638/11701/spbu20.2017.111.

\section{ЗАМЕТКА О ПИШУЩИХ И СТРАНСТВУЮЩИХ ФИЛОСОФАХ}

Фабия Нейербург

В письме Фалеса Ферекиду, которое содержится в жизнеописании Фалеса у Диогена Лаэртского, читателя останавливает необычное разведение двух типов мудрецов: одни философы, подобно туристам наших дней, путешествуют по миру и при этом ничего или почти ничего не пишут, тогда как другие посвящают себя своим трудам, однако никогда не путешествуют. Действительно

\footnotetext{
7 Siehe hierzu die umfassende Studie von O'Sullivan 2011, insbes. 77-96.

${ }^{8}$ Bevor die theoria im Sinne einer Kontemplation zu einem zentralen Begriff der Philosophie wurde, bezeichnet sie ursprünglich die offizielle Gesandtschaft an Kultorte und Feste. Schon bei Herodot wird dieser religiöse Aspekt des Wortes jedoch teilweise abgelegt, die theoria wird ein eher säkulares Reiseerlebnis, auch wenn eine gewisse Ambivalenz bestehen bleibt. Die komplexe Entwicklung vor allem des vorphilosophischen theoria-Begriffes nachzuzeichnen versucht Rausch 1982, wobei sie zurecht das Motiv der Reise für die weitere Evolution des Begriffes betont (42f.). Maria Sassi nennt das Reisemotiv geradezu eine „Scharnierstelle" zwischen der sakralen und philosophischen Bedeutung der theoria, Sassi 1991, 22. Nightingale 2004, unterscheidet verschiedene Formen der vorphilosophischen theoria, die sich durchaus überlappen. Die Entstehung der philosophischen theoria leitet sie im Unterschied zu Rausch und Sassi direkt von der sakralen theoria und dem ihr immanenten „Schauen“ religiöser Festspiele ab (63-71).
} 
ли движение, то есть любое перемещение в пространстве, включая прогулки, несовместимо с жизненной установкой истинного философа - согласно гипотезе Сильвии Монтильо? Пересматривая релевантный текст, автор настоящей заметки задается целью понять, имеет ли указанная несовместимость какое-то отношение к предполагаемому Фалесом разграничению философического писательства и философских путешествий. Внимательное прочтение показывает, что идея Фалеса состоит в противопоставлении двух видов «созерцания»: более традиционная theoria - путешествие с целью приобретения знания - контрастирует с философской, усвоенной и развитой греческими философами IV в. Библиогр. 8 назв.

Ключевые слова: Диоген Лаэртский, Фалес, Ферекид, путешествия философов.

Received 20.02.2017

Final version received 03.05.2017 